\title{
Numerical Study on Transonic Flow with Local Occurrence of Non-Equilibrium Condensation
}

\author{
Shigeru Matsuo ${ }^{1}$, Kazuyuki Yokoo ${ }^{2}$, Junji Nagao ${ }^{2}$, Yushiro Nishiyama ${ }^{2}$, \\ Toshiaki Setoguchi ${ }^{3}$, Heuy Dong $\mathrm{Kim}^{4}$, Shen $\mathbf{Y u}^{5}$ \\ ${ }^{1}$ Department of Advanced Technology Fusion, Saga University, Saga, Japan \\ ${ }^{2}$ Graduate School of Science \& Engineering, Saga University, Saga, Japan \\ ${ }^{3}$ Institute of Ocean Energy, Saga University, Saga, Japan \\ ${ }^{4}$ School of Mechanical Engineering, Andong National University, Andong, Korea \\ ${ }^{5}$ Institute of Engineering Thermophysics, Chinese Academy of Science, Beijing, China \\ Email: matsuo@me.saga-u.ac.jp
}

Received May 24, 2013; revised June 1, 2013; accepted June 8, 2013

Copyright (C) 2013 Shigeru Matsuo et al. This is an open access article distributed under the Creative Commons Attribution License, which permits unrestricted use, distribution, and reproduction in any medium, provided the original work is properly cited.

\begin{abstract}
Characteristics of transonic flow over an airfoil are determined by a shock wave standing on the suction surface. In this case, the shock wave/boundary layer interaction becomes complex because an adverse pressure gradient is imposed by the shock wave on the boundary layer. Several types of passive control techniques have been applied to shock wave/boundary layer interaction in the transonic flow. Furthermore, possibilities for the control of flow fields due to non-equilibrium condensation have been shown so far, and in this flow field, non-equilibrium condensation occurs across the passage of the nozzle and it causes the total pressure loss in the flow field. However, local occurrence of non-equilibrium condensation in the flow field may change the characteristics of total pressure loss compared with that by non-equilibrium condensation across the passage of flow field and there are few for researches of locally occurred non-equilibrium condensation in a transonic flow field. The purpose of this study is to clarify the effect of locally occurred non-equilibrium condensation on the shock strength and total pressure loss on a transonic internal flow field with circular bump. As a result, it was found that shock strength in case with local occurrence of non-equilibrium condensation is reduced compared with that of no condensation. Further, the amount of increase in the total pressure loss in case with local occurrence of non-equilibrium condensation was also reduced compared with that by non-equilibrium condensation across the passage of flow field.
\end{abstract}

Keywords: Compressible; Transonic; Shock Wave; Non-Equilibrium Condensation; Simulation

\section{Introduction}

A shock wave standing on the suction surface of airfoils determines the characteristics of transonic flow field. In this case, the shock wave imposes an adverse pressure gradient on the boundary layer and it makes the shock wave/boundary layer interaction complex. Several types of passive control techniques have been proposed to control the shock wave/boundary layer interaction in the transonic flow field. For instance, Bahi et al. [1] and Raghunathan [2] described that a porous wall and cavity system that applied at the foot of the shock wave was effective in decreasing undesirable adverse pressure gradient of the shock wave/boundary layer interaction. However, this control method essentially leads to large viscous losses caused by the porous walls. These losses may be greater than the control benefit of the shock wave. Thus, the method cannot be generalized as an effective method. In order to overcome the above demerits, Saida et al. [3] proposed several techniques and showed that the porous wall with a cavity and vortex generator to the shock wave/boundary layer interaction was effective method to reduce the wave drag and suppress the development of the boundary layer. Further, Raghunathan [4] and O'Rourke et al. [5] reported that the passive control using the porous wall with a cavity and vortex control jets upstream of porous wall might be effective control method for the shock position and pressure gradient.

Furthermore, the control method using non-equilibrium condensation has been proposed by several re- 
searches (Wegener et al. [6], Matsuo et al. [7], Sislian [8], Setoguchi et al. [9], Matsuo et al. [10], Tanaka et al. [11]). As is evident from these researches, it is known that non-equilibrium condensation can reduce the development of boundary layer behind the shock wave. In the flow fields, non-equilibrium condensation occurs across the passage of the nozzle and it causes the total pressure loss in the flow field. However, local occurrence of non-equilibrium condensation in the flow field may change the characteristics of total pressure loss compared with that by non-equilibrium condensation across the passage of flow field. However, there are few for researches of locally occurred non-equilibrium condensation in a transonic flow field.

The objectives in the present study is to clarify the effect of locally occurred non-equilibrium condensation on the shock strength and total pressure loss in a transonic internal flow field with a circular bump.

\section{CFD Analysis}

\section{Governing Equations}

Following assumptions were used in the present study; both velocity slip and temperature difference do not exist between condensate particles and gas mixture, and the effect of the condensate particles on pressure is neglected. The governing equation, i.e., the unsteady 2D compressible Navier-Stokes equations that were combined with nucleation rate, a droplet growth and diffusion equations (Bird et al. [12], Hirschfelder [13]) written in

$$
\begin{aligned}
& \frac{\partial}{\partial t} \int_{\Omega} \boldsymbol{Q} \mathrm{d} \Omega+\int_{\partial \Omega} \boldsymbol{H} \mathrm{d} S \\
& =\frac{1}{R e} \int_{\partial \Omega} \boldsymbol{R} \mathrm{d} S+\int_{\Omega} \boldsymbol{I} \mathrm{d} \Omega+\int_{\Omega} \boldsymbol{S} \mathrm{d} \Omega
\end{aligned}
$$

where

$$
\begin{aligned}
& \boldsymbol{Q}=\frac{1}{J}\left[\begin{array}{c}
\rho \\
\rho u \\
\rho v \\
E_{t} \\
\rho k \\
\rho g \\
\rho n \\
\rho_{1}
\end{array}\right], \boldsymbol{H}=\frac{1}{J}\left[\begin{array}{c}
\rho U \\
\rho u U+n_{x} p \\
\rho v U+n_{y} p \\
\left(E_{t}+p\right) U \\
\rho k U \\
\rho \omega U \\
\rho g U \\
\rho n U \\
\rho_{1} U
\end{array}\right], \boldsymbol{R}=\left[\begin{array}{c}
0 \\
\tau_{x x} n_{x}+\tau_{x y} n_{y} \\
\tau_{y x} n_{x}+\tau_{y y} n_{y} \\
f_{x} n_{x}+f_{y} n_{y} \\
\left(\mu_{l}+\sigma^{*} \frac{\rho k}{\omega}\right)\left(\frac{\partial k}{\partial x} n_{x}+\frac{\partial k}{\partial y} n_{y}\right) \\
\left(\mu_{l}+\sigma \frac{\rho k}{\omega}\right)\left(\frac{\partial \omega}{\partial x} n_{x}+\frac{\partial \omega}{\partial y} n_{y}\right) \\
0 \\
0 \\
n_{x}\left(\rho_{1} \Delta_{1} \frac{\partial c_{1}}{\partial x}\right)+n_{y}\left(\rho \Delta_{1} \frac{\partial c_{1}}{\partial y}\right)
\end{array}\right] \\
& \boldsymbol{I}=\frac{1}{J}\left[\begin{array}{c}
0 \\
0 \\
0 \\
0 \\
\alpha \frac{\omega}{k} P_{k} \frac{1}{R_{e}}-\beta \rho \omega^{2} R_{e}+\sigma_{\mathrm{d}} \frac{\rho}{\omega}\left(\frac{\partial k}{\partial x} \frac{\partial \omega}{\partial x}+\frac{\partial k}{\partial y} \frac{\partial \omega}{\partial y}\right) R_{e} \\
0 \\
0 \\
0
\end{array}\right], \quad \boldsymbol{S}=\frac{1}{J}\left[\begin{array}{c}
0 \\
0 \\
0 \\
0 \\
0 \\
0 \\
\frac{4}{3} \pi \rho_{l}\left(r_{c}^{3} I_{F}+3 \rho n r^{2} \frac{\mathrm{d} r}{\mathrm{~d} t}\right) \\
I_{F} \\
0
\end{array}\right]
\end{aligned}
$$

In Equation (1), $\boldsymbol{Q}$ is conservative vector, $\boldsymbol{H}$ is inviscid flux vector and $\boldsymbol{R}$ is viscous flux vectors. $\boldsymbol{I}$ and $\boldsymbol{S}$ are the source terms corresponding to turbulence and condensa- tion, respectively. $\tau_{x x}, \tau_{x y}, \tau_{y x}$ and $\tau_{y y}$ are components of viscous shear stress.

In Equation (2), 


$$
\begin{aligned}
& f_{x_{j}}=n_{x}\left(q_{x}+u \tau_{x x}+v \tau_{x y}\right)+n_{y}\left(q_{y}+u \tau_{y x}+v \tau_{y y}\right) \\
& \tau_{c 1}=n_{x}\left(\rho_{1} \Delta_{1} \frac{\partial c_{1}}{\partial x}\right)+n_{y}\left(\rho \Delta_{1} \frac{\partial c_{1}}{\partial y}\right) \\
& q_{x_{j}}=\frac{1}{\gamma-1}\left(\frac{\mu}{P r}+\frac{\mu_{\mathrm{t}}}{P r_{\mathrm{t}}}\right) \frac{\partial T}{\partial x_{\mathrm{j}}}+\rho\left(h_{1} \Delta_{1} \frac{\partial c_{1}}{\partial x_{\mathrm{j}}}+h_{2} \Delta_{2} \frac{\partial c_{2}}{\partial x_{\mathrm{j}}}\right) \\
& f_{k}=\mu+\sigma * \frac{\rho k}{\omega}, f_{\omega}=\mu+\sigma \frac{\rho k}{\omega} \\
& f_{\mathrm{H}}=\alpha \frac{\omega}{k} P_{k}-\beta \rho \omega^{2}+\sigma_{\mathrm{d}} \frac{\rho}{\omega}\left(\frac{\partial k}{\partial x} \frac{\partial \omega}{\partial x}+\frac{\partial k}{\partial y} \frac{\partial \omega}{\partial y}\right) \\
& P_{k}=2 \mu_{\mathrm{t}}\left[\left(\frac{\partial u}{\partial x}\right)^{2}+\frac{1}{2}\left(\frac{\partial v}{\partial x}+\frac{\partial u}{\partial y}\right)+\left(\frac{\partial v}{\partial y}\right)^{2}\right] \\
& \mu_{\mathrm{t}}=\frac{\rho k}{\tilde{\omega}}, \quad \tilde{\omega}=\max \left\{\sqrt{\frac{2 S_{\mathrm{i}, \mathrm{j}} \bar{S}_{\mathrm{i}, \mathrm{j}}}{\beta *}}\right\} \\
& \bar{S}_{\mathrm{i}, \mathrm{j}}=S_{\mathrm{i}, \mathrm{j}}-\frac{1}{3} \frac{\partial u_{\mathrm{k}}}{\partial x_{\mathrm{k}}}, S_{\mathrm{i}, \mathrm{j}}=\frac{1}{2}\left(\frac{\partial u_{\mathrm{i}}}{\partial x_{\mathrm{j}}}+\frac{\partial u_{\mathrm{j}}}{\partial x_{\mathrm{i}}}\right)
\end{aligned}
$$

The density of gas mixture is calculated by the sum of density of vapor $\left(\rho_{1}\right)$ and dry $\operatorname{air}\left(\rho_{2}\right)$;

$$
\rho=\rho_{1}+\rho_{2}
$$

The mass fraction can be given as

$$
c_{\mathrm{i}}=\frac{\rho_{\mathrm{i}}}{\rho}
$$

In Equations (4)-(6), $\Delta_{1}$ and $\Delta_{2}$ are effective diffusivities of vapor and dry air, respectively. The closure coefficients are,

$$
\begin{aligned}
& \beta=0.0708, \beta^{*}=\frac{9}{100}, \sigma=\frac{1}{2}, \\
& \sigma^{*}=\frac{3}{5}, \operatorname{Pr}_{\mathrm{t}}=\frac{7}{8} \\
& \sigma_{\mathrm{d}}=\left\{\begin{array}{l}
0, \quad \frac{\partial k}{\partial x_{\mathrm{j}}} \frac{\partial \omega}{\partial x_{\mathrm{j}}} \leq 0 \\
\sigma_{\mathrm{d} 0}, \frac{\partial k}{\partial x_{\mathrm{j}}} \frac{\partial \omega}{\partial x_{\mathrm{j}}} \geq 0 \quad\left(\sigma_{\mathrm{d} 0}=\frac{1}{8}\right)
\end{array}\right.
\end{aligned}
$$

The governing equation system are non-dimensionalized with reference values at the reservoir condition. Implicit upwind relaxation scheme (Furukawa et al. [14]) is used to solve the governing equations. The equations were discretized in time using the Euler implicit method and in space using a cell-centered finite volume method with a quadrilateral structured cell system. A third-order TVD scheme with MUSCL (Yee [15]) was used to discretize the spatial derivatives, and second-order central difference scheme for the viscous term. In the solver, the relaxation was performed with a point Gauss-Seidel technique. To close the governing equations, $k-\omega$ model (Wilcox [16]) was employed in computations.

\section{Computational Conditions}

Figure 1 shows a computational domain of the transonic flow field and boundary condition. The test section has a height of $H=60 \mathrm{~mm}$ at the inlet and exit. The radius of circular arc of the nozzle is $R=100 \mathrm{~mm}$ and the height of nozzle throat $H^{*}$ is $56 \mathrm{~mm}$. The region upstream of the nozzle was separated into dry air and moist air regions by plate. Thickness of the plate is $0 \mathrm{~mm}$. Furthermore, working gas (dry air, moist air) in a tank flows into the main flow from the leading edge of the circular bump through the narrow passage $(\mathrm{d}=3 \mathrm{~mm})$

Table 1 shows initial conditions used in the present calculation. Total pressure $p_{0}$ and temperature $T_{0}$ at stagnation point upstream of the nozzle are $102 \mathrm{kPa}$ and 287 $\mathrm{K}$, respectively.

The working gases of upper and lower sides of the plate are dry and moist airs, respectively. Values of $Y / H$

$$
\left.\left[\begin{array}{l}
T_{0}=287 \mathrm{~K} \\
p_{0}=102 \mathrm{kPa}
\end{array}\right] \quad \begin{array}{c}
u=v=0 \\
\frac{d T}{d y}=0, \frac{d p}{d y}=0 \\
g=0
\end{array}\right]
$$

Out flow

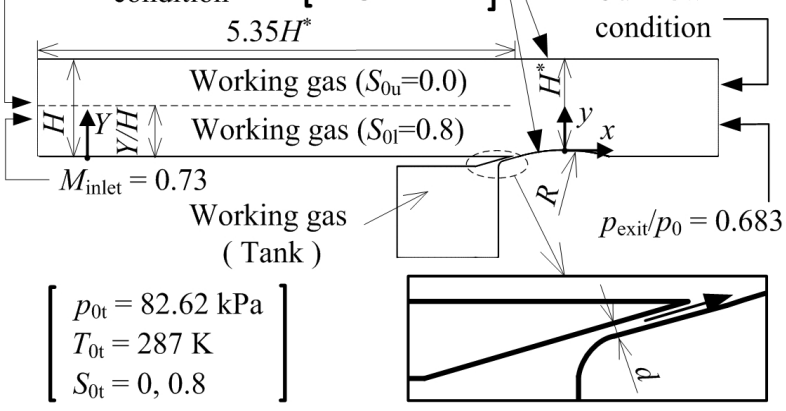

Figure 1. Computational domain and boundary condition.

Table 1. Initial conditions.

\begin{tabular}{ccccc}
\hline & & & & $T_{0}=287 \mathrm{~K}$ \\
\hline Case No. & $p_{0 \mathrm{t}} / p_{0}$ & $\mathrm{~d}[\mathrm{~mm}]$ & $Y / H$ & $\begin{array}{c}\text { Initial degree } \\
\text { of supersaturation }\end{array}$ \\
Case 1-D & & & 0 & $S_{0 \mathrm{u}}=S_{01}=0$ \\
Case 1-M-A & - & 0 & 1 & \\
Case 1-M-B & & & 0.0625 & $S_{0 \mathrm{u}}=0 S_{01}=0.8$ \\
Case 1-M-C & & & 0.0313 & \\
Case 2-D & & & & $S_{0 \mathrm{t}}=0$ \\
Case 2-M3 & 0.81 & 3 & 0 & $S_{0 \mathrm{t}}=0.8$ \\
\hline
\end{tabular}


which shows the plate position, are 0 (Case 1-D, 2-D3, 2-M3), 1.0 (Case 1-M-A), 0.0625 (Case 1-M-B) and 0.0313 (Case 1-M-C). Furthermore, stagnation pressure $p_{0 \mathrm{t}}$ in the rank is $82.62 \mathrm{kPa}$ and working gas in the tank is dry air (Case 2-D3) or moist air (Case 2-M3). Initial degree of supersaturation $\left(S_{0}, S_{0 t}\right)$ of moist air is 0.8 .

The number of grids is $450 \times 228$. The adiabatic noslip wall was used as boundary condition. The boundary conditions of inlet and exit were fixed at initial condition and out flow condition, respectively. Condensate mass fraction $g$ was set at $g=0$ on the wall.

\section{Result and Discussion}

Figure 2 shows schlieren photographs obtained by experiment (Figures 2(a) and (c)) and computer schlieren pictures (Figures 2(b) and (d)). In Figures 2(a) and (b), working gas is dry air (Experiment: $S_{0}=0.18$, Simulation: $\left.S_{0}=0\right)$ and moist air $\left(S_{0}=0.5\right)$ in Figures 2(c) and (d). Flow direction is left to right. As seen from these figures, shock wave is observed on the circular arc bump. In the case of dry air (Figures 2(a) and (b)), the shock wave is clearly visible compared with that of moist air (Figures 2(c) and (d)).

Figures 3(a) and (b) show static pressure distributions on the lower wall in cases of dry air and moist air, respectively. In each figure, comparison between the experimental and simulated static pressure distributions are shown and it is found from these figures that simulated results agree well with experimental results.

Figure 4 shows time-averaged contour maps of Mach number (line) and condensate mass fraction $g$ (gray) for all cases. As seen from Figures 4(b)-(d) and (f), condensate mass fraction (condensate droplet) begins to in-

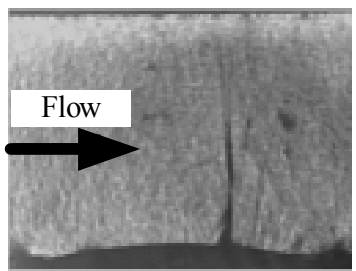

(a)

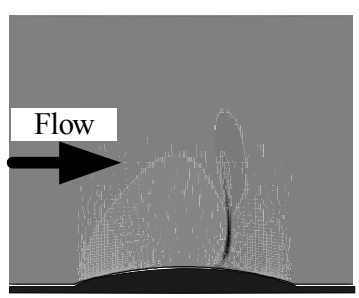

(b)

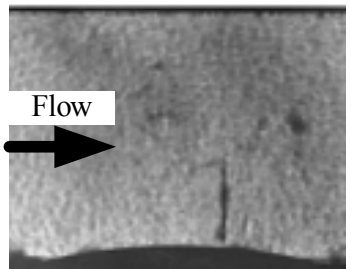

(c)

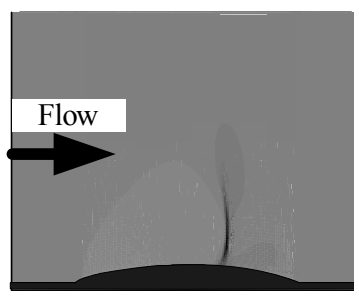

(d)
Figure 2. Comparison between the experiment and simulated results. (a) Experiment $\left(S_{0}=0.18\right)$; (b) Simulation $\left(S_{0}\right.$ $=0.0$ ); (c) Experiment $\left(S_{0}=0.5\right)$; (d) Simulation $\left(S_{0}=0.5\right)$.

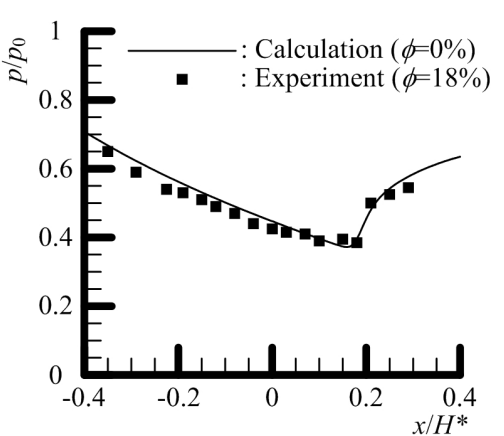

(a)

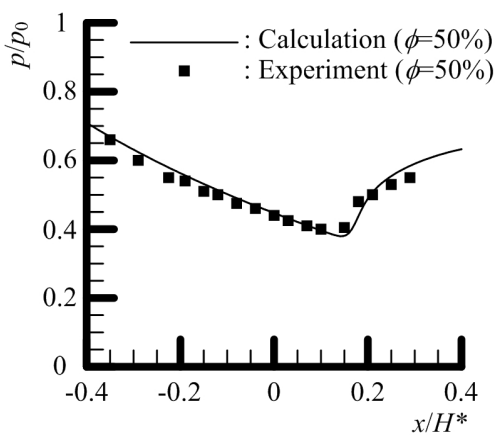

(b)

Figure 3. Comparison between the experimental and simulated static pressure distributions on the lower wall. (a) Dry air; (b) Moist air.

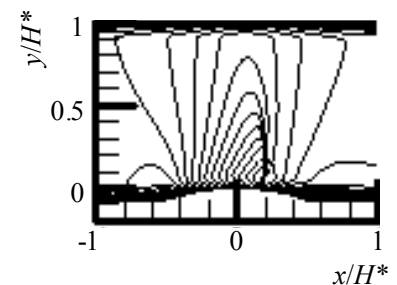

(a)

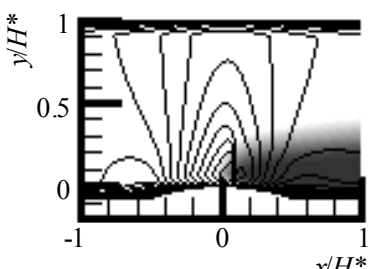

(b)

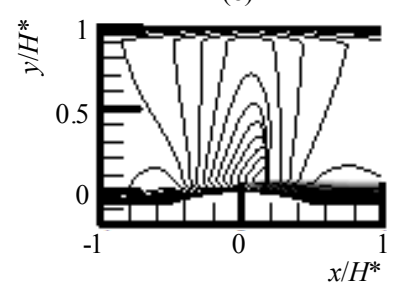

(c)

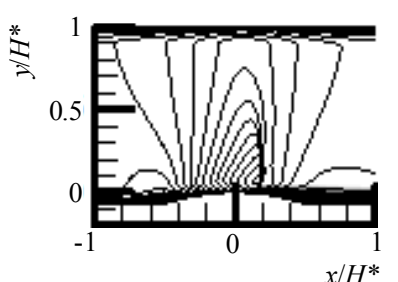

(d)

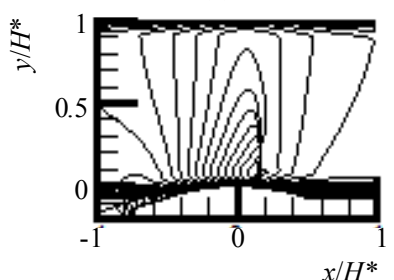

(e)

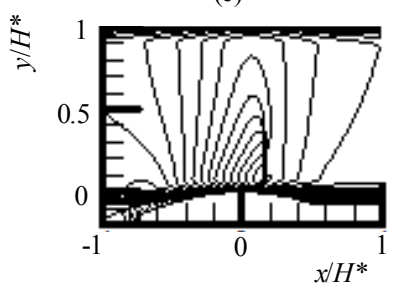

(f)
Figure 4. Contour maps of Mach number (line) and condensate mass fraction (Grey). (a) Case 1-D; (b) Case 1-M-A; (c) Case 1-M-B; (d) Case 1-M-C; (e) Case 2-D3; (f) Case 2-M3. 
crease along the bump wall side upstream of the shock wave and distributes over downstream region, and it expands in the positive direction of $y$-axis in order of Cases 1-M-C, 1-M-B and 1-M-A. Furthermore, for Cases 1-MA (Figure 4(b)) to 2-M3 (Figure 4(f)), the height of adiabatic shock wave seems to become small compare with that for Case 1-D (Figure 4(a)).

Figure 5 shows distributions of shock strength $(\phi=$ $\left.p_{2} / p_{1}\right)$ in the direction of $y$-axis for all cases. As seen from this figure, shock strength for Case 1-D is the largest in all cases and it decreases in order of Case 1-M-C, $1-\mathrm{M}-\mathrm{B}$ and 1-M-A. However, there is no difference of the strength between Cases 1-M-C and 1-M-B, as well as the difference between Cases 2-D3 and 2-M3. It is found from this result that the strength is changed by occurrence region of non-equilibrium condensation.

Figure 6 shows distributions of integrated total pressure loss $\beta$ in the direction of $x$-axis. Integrated total pressure loss is calculated from following equation:

$$
\beta=\int_{\text {Lower wall }}^{\text {Upper wall }}\left(1-\frac{p_{01}}{p_{0}}\right) \mathrm{d} y
$$

where $p_{01}$ and $p_{0}$ indicate local and stagnation total pressures, respectively.

From this figure, integrated total pressure losses of Cases 1-M-A, 1-M-B and 1-M-C begin to deviate from the distribution of Case 1-D at the position just upstream of the shock wave. It is considered that this is due to an increase of entropy by non-equilibrium condensation occurred upstream of the shock wave. Integrated total pressure losses for Cases 1-M-C is small compared with that of 2-D3 and 2-M3. Further, integrated total pressure losses of Cases 2-D3 and 2-M3 begin to deviate from the distribution of Case 1-D at the position close to the leading edge of the circular bump. This is due to effect of

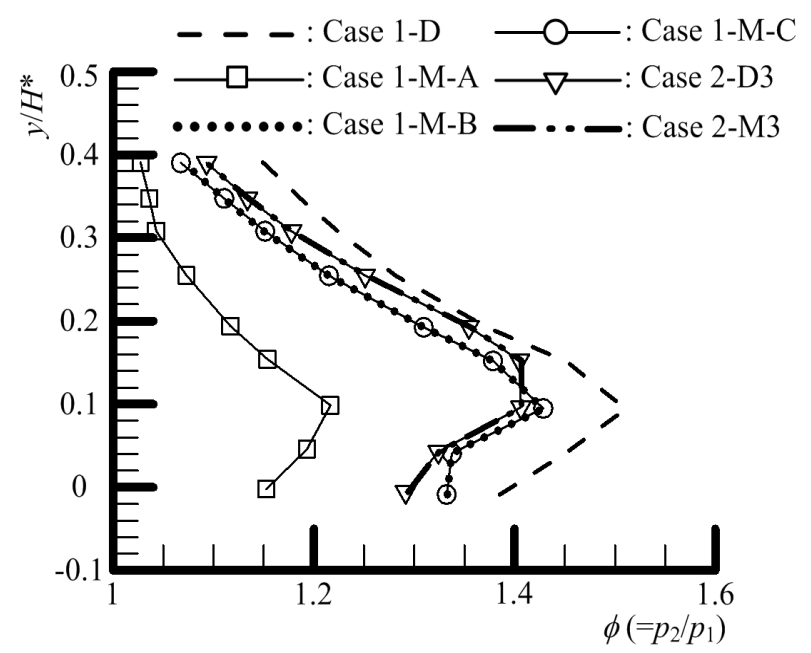

Figure 5. Distributions of shock strength $\phi$. blowing of the flow through the narrow passage. Based on the results in Figures $\mathbf{5}$ and $\mathbf{6}$, it is considered from the point of view of energy loss that the flow of Case $1-\mathrm{M}-\mathrm{C}$ is the most effective in all cases.

Figure 7 shows time-averaged distributions of displacement thickness $\delta^{*} / H^{*}$ for all cases. The abscissa is the distance $x / H^{*}$ from throat, and the ordinate is displacement thickness $\delta^{*} / H^{*}$. As is evident from this figure, displacement thickness behind the shock wave for Case 1-M-A is the smallest in all cases and in Cases 2-D3 and 2-M3, it is high compared with that for other cases in $-0.5 \leq x / H^{*} \leq 0.5$. From these results, it is found that the development of boundary layer is dependent on how the non-equilibrium condensation occurs.

\section{Conclusions}

A numerical study has been made to investigate the effect of locally occurred non-equilibrium condensation on a transonic flow field with a circular arc bump. The

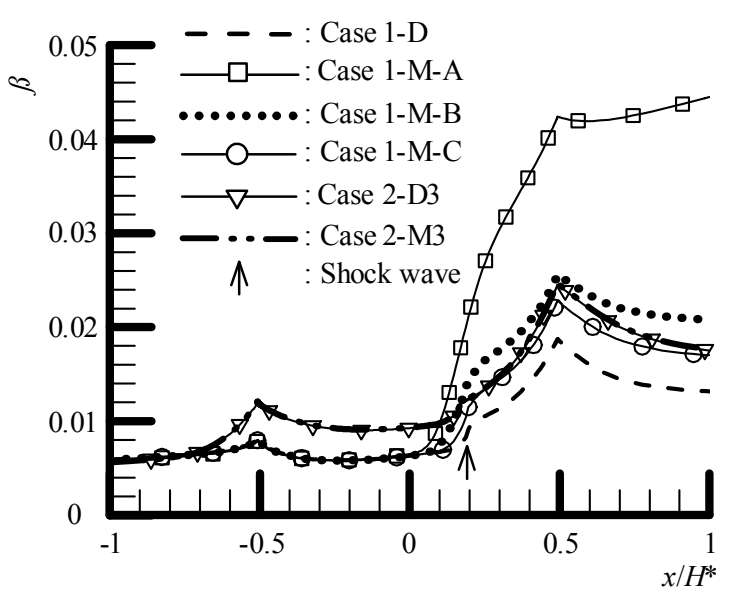

Figure 6. Distributions of integrated total pressure loss $\beta$.

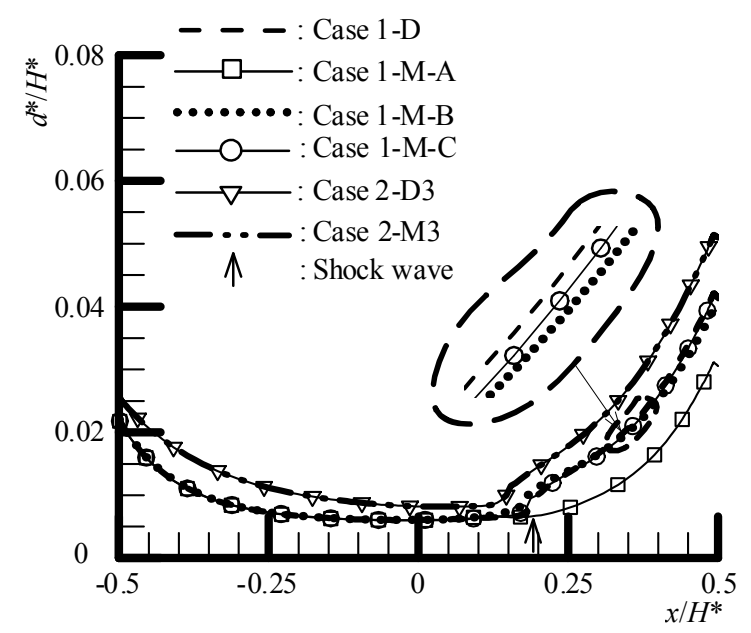

Figure 7. Time-averaged distributions of displacement thickness $\delta^{*} / H^{*}$. 
results obtained are summarized as follows:

1) Locally occurred non-equilibrium condensation on the transonic bump model weakened the shock strength.

2) Compared with the case of occurrence of non-equilibrium condensation across the passage of the nozzle, locally occurred non-equilibrium condensation could reduce the total pressure loss downstream of the shock wave.

3) The development of boundary layer was dependent on how the non-equilibrium condensation occurs.

\section{REFERENCES}

[1] L. Bahi, J. M. Ross and T. Nagamatsu, "Passive Shock Wave/Boundary Layer Control for Transonic Airfoil Drag Reduction," AIAA 21st Aerospace Sciences Meeting, Nevada, 10-13 January 1983, AIAA-83-0137, 12 pp.

[2] S. Raghunathan, "Passive Control of Shock-Boundary Layer Interaction," Progress in Aerospace Sciences, Vol. 25, No. 3, 1998, pp. 271-296. doi:10.1016/0376-0421(88)90002-4

[3] N. Saida and Y. Tomizuka, "Passive Control of Oblique Shock/Boundary Layer Interaction," Journal of the Japan Society for Aeronautical and Space Sciences, Vol. 50, No. 581, 2002, pp. 223-230. doi:10.2322/jisass.50.223

[4] S. R. Raghunathan, M. O'Rourke, J. K. Watterson, R. K. Cooper and E. Benard, "Passive Vortex Control Jets for Shock Boundary Layer Interactions," AIAA 17th Applies Aerodynamics Conference, Virginia, 28 June-2 July 1999 , AIAA-99-3196.

[5] M. J. O'Rourke, M. Healy and S. R. Raghunathan, "Computational Experiment Investigating Passive Vortex Control Jets for Shock Boundary Layer Interactions," $31 s t$ AIAA Fluid Dynamics Conference \& Exhibit, California, 11-14 June 2001, AIAA-2001-3028.

[6] P. P. Wegener and L. M. Mach, "Condensation in Supersonic Hypersonic Wind Tunnels," Advances in Applied Mechanics, Vol. 5, 1958, pp. 307-447. doi:10.1016/S0065-2156(08)70022-X

[7] K. Matsuo, S. Kawagoe and K. Sonoda, "Studies of Condensation Shock Waves (Part 1, Mechanism of their Formulation)," Bulletin of JSME, Vol. 28, No. 241, 1985, pp. 1416-1422. doi:10.1299/jsme1958.28.1416

[8] J. P. Sislian, "Condensation of Water Vapour with or without a Carrier Gas in a Shock Tube," UTIAS Report No. 201, Institute for Aerospace Studies, University of Toronto, Toronto, 1975.

[9] T. Setoguchi, S. Matsuo and S. Yu, "Effect of Nonequilibrium Homogeneous Condensation on Flow Fields in a Supersonic Nozzle," Journal of Thermal Science, Vol. 6, No. 2, 1997, pp. 90-96. doi:10.1007/s11630-997-0022-5

[10] S. Matsuo, T. Setoguchi and S. Yu, "Effect of Nonequilibrium Condensation of Moist Air on the Boundary Layer in a Supersonic Nozzle," Journal of Thermal Science, Vol. 6, No. 4, 1997, pp. 260-272. doi:10.1007/s11630-997-0005-6

[11] M. Tanaka, S. Matsuo and T. Setoguchi, "Passive Control of Transonic Flow Fields with Shock Wave Using NonEquilibrium Condensation and Porous Wall," Journal of Thermal Science, Vol. 2, No. 12, 2003, pp. 128-131. doi:10.1007/s11630-003-0053-5

[12] R. B. Bird, W. E. Stewart and E. N. Lightfoot, "Transport Phenomena," Wiley, New York, 1960.

[13] J. O. Hirschfelder, C. F. Curtiss and R. B. Bird, "Molecular Theory of Gases and Liquids," Wiley, New York, 1954. doi:10.1016/0016-0032(55)91080-2

[14] M. Furukawa, T. Nakano and M. Inoue, "Unsteady Navier-Stokes Simulation of Transonic Cascade Flow Using an Unfactored Implicit Upwind Relaxation Scheme with Inner Iterations," Journal of Turbomachinery, Vol. 114, No. 3, 1992, pp. 599-606. doi:10.1115/1.2929184

[15] H. C. Yee, "A Class of High-Resolution Explicit and Implicit Shock Capturing Methods," NASA, Washington DC, 1989.

[16] D. C. Wilcox, "Formulation of the $k$-Turbulence Model Revisited," AIAA Journal, Vol. 46, No. 11, 2008, pp. 2823-2838. doi:10.2514/1.36541 